\title{
Sex influences the association between haemostasis and the extent of lung lesions in tuberculosis
}

Wenling Tan ${ }^{1 \dagger}$, Adiilah K Soodeen-Lalloo ${ }^{2 \dagger}$, Yue Chu', Weijie Xu ${ }^{3}$, Fengfang Chen ${ }^{1,4}$, Jie Zhang ${ }^{5}$, Wei Sha ${ }^{1,6}$, Jin Huang ${ }^{4}$, Guanghong Yang ${ }^{4}$, Lianhua Qin ${ }^{1}$, Jie Wang ${ }^{1}$, Xiaochen Huang ${ }^{1}$, Jingyun Shi ${ }^{2^{*}}$ and Yonghong Feng ${ }^{1,6^{*}}$

\begin{abstract}
Background: Worldwide tuberculosis (TB) reports show a male bias in morbidity; however, the differences in pathogenesis between men and women with TB, as well as the mechanisms associated with such differences, are poorly investigated. We hypothesized that comparison of the degree of lung injury and clinical indices of well-matched men and women with newly diagnosed TB, and statistical analysis of the correlation between these indices and the extent of lung lesions, can provide insights into the mechanism of gender bias in TB.

Methods: We evaluated the acid-fast bacilli grading of sputum samples and compiled computed tomography (CT) data of the age-matched, newly diagnosed male and female TB patients without history of smoking or comorbidities. Inflammatory biomarker levels and routine haematological and coagulation-associated parameters were compared. Binary logistic regression analysis was used to define the association between the indices and lung lesions, and the influence of sex adjustment.
\end{abstract}

Results: Women with TB have a longer delay in seeking healthcare than men after onset of the TB-associated symptoms. Men with TB have significantly more severe lung lesions (cavities and healing-associated features) and higher bacterial counts compared to women with TB. Scoring of the CT images before and after anti-TB treatment showed a faster response to therapy in women than in men. Coagulation- and platelet-associated indices were in models from multivariate regression analysis with groups of males or females with TB or in combination. In univariate regression analysis, lower lymphocyte counts were associated with both cavity and more bacterial counts, independent of sex, age and BMI. The association of international normalized ratios (INR), prothrombin times (PTs), mean platelet volumes (MPVs) and fibrinogen (FIB) level with lung lesions was mostly influenced by sex adjustment.

Conclusions: Sex influences the association between haemostasis and extent of TB lung lesions, which may be one mechanism involved in sex bias in TB pathogenesis.

Keywords: Tuberculosis, Gender bias, Coagulation, Platelet

\footnotetext{
* Correspondence: shijingyun89179@126.com; feng_yonghong@hotmail.com

${ }^{+}$Wenling Tan and Adiilah K Soodeen-Lalloo contributed equally to this work.

2Department of Radiology, Shanghai Pulmonary Hospital, Tongji University

School of Medicine, Shanghai 200433, China

${ }^{1}$ Shanghai Key Laboratory of Tuberculosis, Shanghai Pulmonary Hospital,

Tongji University School of Medicine, 507 Zhengmin Road, Shanghai 200433,

China

Full list of author information is available at the end of the article
}

(c) The Author(s). 2018 Open Access This article is distributed under the terms of the Creative Commons Attribution 4.0 International License (http://creativecommons.org/licenses/by/4.0/), which permits unrestricted use, distribution, and reproduction in any medium, provided you give appropriate credit to the original author(s) and the source, provide a link to the Creative Commons license, and indicate if changes were made. The Creative Commons Public Domain Dedication waiver (http://creativecommons.org/publicdomain/zero/1.0/) applies to the data made available in this article, unless otherwise stated. 


\section{Background}

It is estimated that one third of the world's population is infected with Mycobacterium tuberculosis (Mtb.), the pathogen that causes tuberculosis (TB). TB was directly responsible for approximately 1.7 million deaths in 2016 and remains one of the top 10 causes of death worldwide despite the availability of drug treatment [1]. Epidemiological surveys have indicated that men tend to be affected more than women, with a man to woman ratio close to 2 as per the worldwide case notification rates [1]. The degree of male bias varies by geographic location and by year, but the overall trend is clear [2].

While disagreement exists on whether socioeconomic and cultural factors may create barriers to accessing healthcare which hence cause undernotification in women, particularly in developing countries [3-5], in recent years, however, increasing numbers of studies have indicated that biological mechanisms may actually account for a significant part of the difference between men and women in susceptibility to infection, including TB [6-8]. Consistently, with C57BL/6 and Balb/c mouse models, previous researches have demonstrated that infection with $M t b$. resulted in exacerbated pulmonary pathology and increased morbidity and mortality in male mice compared to females [9, 10]. The study showed that female and castrated male mice exhibited significant higher inflammation (higher TNF- $\alpha$, IFN $\gamma$, IL12, iNOS and IL17) in all lung compartments than non-castrated males during the first month of infection [10]. In a genotyping study based on a South African population, Salie et al. found sex-specific associations for TLR8 polymorphisms with susceptibility to TB [11]. Nevertheless, evidence for the sex bias in TB pathogenesis based on clinical observation is still scarce, and the underlying mechanisms are still little known.

In this study, we compared the bacterial counts in sputum and the severity of lung lesions on computed tomography (CT) between two groups of age-matched men and women with newly diagnosed TB. We hypothesize that comparison of the biochemical and immunological indices between the groups followed by regression analyses, with and without adjustment for sex, will reveal the indices involved in sex bias in TB-associated lung lesions.

\section{Methods}

\section{Study subjects}

We included primary pulmonary TB patients from Shanghai Pulmonary Hospital (SPH) between April 2011 and April 2015. TB was diagnosed based on acid-fast bacilli (AFB) staining and culture; patients whose cultures yielded nontuberculous mycobacteria were excluded from the study. We retrospectively reviewed medical records and excluded patients who had history of any of the following: smoking, excessive alcohol drinking, human immunodeficiency virus (HIV) infection, immunosuppressive drug therapy, hormone therapy, cancer, diabetes, pneumoconiosis, silicosis and hepatitis B and C viruses' infection. In the enrolled 114 pairs of male and female patients matched for age ( \pm 3 years), none of the patients had received or initiated anti-TB drug therapy for more than 1 week before registration at SPH.

Documented data (complete blood count, CBC) from 62 pairs of healthy men (30.2 \pm 6.7 years) and women $(29.8 \pm 6.9$ years) employees in SPH who had health examination at the same period were used as controls.

\section{Review of clinical findings and laboratory tests}

We retrospectively reviewed patient characteristics including age, sex, height, weight, body mass index (BMI), symptoms, cavity and sputum smear grade. Changes in inflammatory biomarker levels and routine haematological and biochemical parameters were also reviewed. All data were obtained from the first medical records during patient admission.

\section{T-SPOT.TB tests and anti-TB IgG antibody detection}

T-SPOT.TB and serum anti-TB IgG antibody detection were carried out using commercially available T-SPOT.TB kits (Oxford Immunotec. Ltd) and ELISA kits based on a dot immunogold filtration assay (DIGFA) with specific membrane antigens from $M t b$. bound to a nitrocellulose filter membrane (Shanghai Aopu Biomed Ltd. Company, China), respectively. Blood samples were collected and peripheral blood mononuclear cells (PBMCs) were prepared within $2 \mathrm{~h}$ as per standard protocol. All procedures, including interpretation of the results, were carried out according to the manufacturer's recommendations. The absolute numbers of spot-forming cells (SFCs) were counted using an Elispot counter (AID, Strasberg, Germany), and the numbers of antigen-specific SFCs in positive T-SPOT.TB assays were plotted following the subtraction of the numbers in the negative control.

\section{AFB grading}

The Auramine $\mathrm{O}$ fluorescent staining method was used to stain the sputa [12]. The grades of the AFB smear-positive samples were reported according to the Standard Procedures for Laboratory Diagnosis of Tuberculosis by the Chinese Anti-Tuberculosis Association as follows [13]: grade $1(1+), 10-99$ bacilli/50 fields; grade 2 (2+), 1-9 bacilli/field; grade 3 (3+), 10-99 bacilli/field; and grade $4(4+), 100$ bacilli/field. The highest grade for each individual within the first week of admittance was selected for analysis. Patients were divided into two groups with low $(1+$ and $2+)$ and high $(3+$ and $4+)$ sputum bacteria counts for binary regression analysis. 


\section{Computed tomography evaluation}

In total, high-resolution computed tomography (HRCT) scans from 94 female and 95 male patients performed within the first week of admittance (T0) were collected. The scans were assessed by two specialists who were blinded to the groups of patients. Lungs were divided into six zones (low, middle and high zones for the left and right lungs), and the presence of abnormalities including nodule, micronodule, cavity, consolidation, parenchymal bands, ground glass opacity and bronchial lesion was noted according to previous reports [14, 15]. The total weighted HRCT score was calculated as HRCT score $\times 100 / 168$ (total score) +40 if cavitation was present.

Descriptive terms used to interpret the CT findings were defined as previously reported [16]. Cavity wall thickness $>3 \mathrm{~mm}$ was defined as thick-walled, and cavity wall thickness $\leq 3 \mathrm{~mm}$ was defined as thin-walled.

\section{Follow-up analysis of the response to anti-TB treatment}

The responses of the patients to standard anti-TB treatment (a standard regimen consisting of isoniazid, rifampin, pyrazinamide and ethambutol (2HRZE/4HRE) according to the National TB Programme) were evaluated by tracking the records of sputum bacterial grades and CT imaging after 1 (T1) and 3 months (T3) of therapy.

In total, records of sputum bacterial counts from 87 females and 79 males were tracked and scored. Thirtyfour female and 24 male patients at T1 and 36 female and 28 male patients at T3 had CT scan records; only 15 female and 7 male patients had complete records of CT scans at $\mathrm{T} 0, \mathrm{~T} 1$ and $\mathrm{T} 3$.

\section{Ethical approval}

This study was conducted in accordance with the amended Declaration of Helsinki and the ethical guidelines of the institutional review board of Tongji University (project approval number 2014fk10). All participants gave written consent for the use of their clinical information for research purposes. Clinical data were anonymized.

\section{Statistical analyses}

We performed $\chi^{2}$ test for categorical variables, Wilcoxon rank-sum test for nominal variables and $t$ tests for continuous variables. To identify the parameters associated with the extent of lung lesions (sputum bacterial counts and cavity), 114 pairs of male and female TB patients with complete records of 48 physiological, haematological and biochemical analyses were chosen for multivariate (male and female groups separately and in combination) and univariate (combination of male and female cases) logistic regression analyses. In univariate regression analysis, the association was adjusted for age/
BMI and sex, separately or in combination, to test the influence of these factors on the association. Statistical significance was determined at $P<0.05$. All analyses were performed using SPSS (version 19, SPSS Inc., Chicago, IL, USA).

\section{Results \\ Patient characteristics}

Although the 114 pairs of age-matched female (35.2 \pm 14.0 years) and male (35.1 \pm 13.9 years) TB patients had significant differences in height and weight, they showed no significant differences in BMI at the time of first registration (F $19.3 \pm 2.4$ versus $M 19.8 \pm 2.6 ; P=0.136$ ). Besides, ratios of cases with $\mathrm{BMI}<18.5$ (malnutrition) were without statistical difference in the two groups ( $\mathrm{F}$ $38.5 \%$ versus $M 31.4 \%, P=0.276$ ). Intriguingly, a significant difference was found between the groups in time elapsed between onset of symptoms and seeking medical care. A higher ratio of men with $\mathrm{TB}$, compared to women with TB, sought healthcare within 1 month of onset of symptoms (32.5\% versus $14.9 \%)$; more than half of the female patients (59.6\%) sought healthcare between 1 and 6 months after onset of symptoms (Table 1).

Higher sputum bacterial counts $(3+$ and $4+)$ were observed in $46.5 \%$ of male patients compared to $25.6 \%$ in female patients. There were no statistical differences between male and female TB patients in the ratios of cases with positive responses in both the anti-TB antibody and the T-SPOT.TB tests (Table 1).

\section{Differential lung lesions and response to anti-TB treatment in male and female patients}

Consistent with the results of the sputum bacterial counts, HRCT scans at T0 showed more severe lung damage in men with TB than in women with TB, represented by more cavitary lesions $(70.5 \%$ versus $37.2 \%, P<$ $0.001)$, as well as healing-associated cicatricial emphysema, parenchymal bands, bronchovascular distortion and pleural thickening (Table 2).

In the follow-up analysis of the overall response to the therapy, the grades of the bacterial loads in sputa showed a faster negative-conversion tendency $(P=0.069)$ in women $(60.9 \%, 53 / 87)$ than in men $(46.8 \%, 37 / 79)$ after 1 month of treatment (Additional file 1: Table S1).

Changes in the HRCT indices reflected detailed responses to standard anti-TB treatment. However, as fewer $\mathrm{CT}$ records (34 females and 24 males at $\mathrm{T} 1$ and $36 \mathrm{fe}-$ males and 28 males at T3) from the enrolled patients could be tracked back to $\mathrm{T} 1$ and $\mathrm{T} 3$ (Additional file 1: Table S2), the changes in CT images from patients with complete $\mathrm{T} 0, \mathrm{~T} 1$ and $\mathrm{T} 3$ records were better to reflect the response to the treatment.

In 15 female and 7 male TB patients with complete HRCT records at T0, T1 and T3, the HRCT scores, 
Table 1 Characteristics of study patients

\begin{tabular}{|c|c|c|c|}
\hline & \multicolumn{2}{|c|}{ Sputum smear-positive TB } & \multirow[t]{2}{*}{$P$ value } \\
\hline & Female $(n=114)$ & Male $(n=114)$ & \\
\hline Age, mean (sd), year & $35.2(14.0)$ & $35.1(13.9)$ & $0.943^{\mathrm{a}}$ \\
\hline Height, $\mathrm{cm}^{\dagger}$ & $160(158-164) n=109$ & $173(170-177) n=105$ & $<0.001^{\mathrm{a}}$ \\
\hline Weight, $\mathrm{kg}^{\dagger}$ & $50(45-55) n=109$ & $60(53-66) n=106$ & $<0.001^{\mathrm{a}}$ \\
\hline $\mathrm{BMl}$, mean (sd), kg/m² & $19.3(2.4) n=109$ & $19.8(2.6) n=105$ & $0.136^{\mathrm{a}}$ \\
\hline $\mathrm{BMI}<18.5$, number $(\%)$ & $42(38.5)(n=109)$ & $33(31.4)(n=105)$ & $0.276^{c}$ \\
\hline \multicolumn{3}{|c|}{ Time elapsed between onset of symptoms and admittance to SPH, months, number (\%) ${ }^{d}$} & $<0.001^{b}$ \\
\hline No symptoms ${ }^{e}$ & $6(5.3)$ & $14(12.3)$ & - \\
\hline$<1$ & $17(14.9)$ & $37(32.5)$ & - \\
\hline $1-6$ & $68(59.6)$ & $49(43.0)$ & - \\
\hline $6-12$ & $12(10.5)$ & $8(7.0)$ & - \\
\hline$>12$ & $11(9.6)$ & $6(5.3)$ & - \\
\hline \multicolumn{3}{|l|}{ Acid-fast bacilli, number (\%) } & $<0.001^{b}$ \\
\hline $1+$ & $56(49.1)$ & $33(28.9)$ & - \\
\hline $2+$ & $29(25.4)$ & $28(24.6)$ & - \\
\hline $3+$ & $23(20.3)$ & $30(26.3)$ & - \\
\hline $4+$ & $6(5.3)$ & $23(20.2)$ & - \\
\hline Anti-TB antibody, number (\%) & $56(49.1)$ & $49(43.0)$ & $0.352^{c}$ \\
\hline T-SPOT.TB, number (\%) & $66(84.62) n=78$ & $73(90.12) n=81$ & $0.295^{c}$ \\
\hline
\end{tabular}

\footnotetext{
TB tuberculosis. Italicized numbers indicated a $P$ value of $<0.05$

${ }^{+}$Data are displayed as medians and interquartile ranges

${ }^{\mathrm{a}} t$ tests

bilcoxon tests

${ }^{c} x^{2}$ tests

${ }^{\mathrm{d}}$ TB-correlated symptoms include cough, expectoration, fever, fatigue, hemoptysis, appetite loss, dyspnea, respiratory distress, insomnia, palpitation and weight loss

'Patients without typical TB symptoms; diagnosed by physical examination
}

which are results of simple addition of each index of the lung pathology in details, showed significant decrease in both male and female patients after 3 months of treatment, with a significant difference between the groups $(P=0.044)$, while only women patients showed a significant decrease in HRCT scores weighted by the presence of cavities (Table 3). Cavity results from accumulating immune response of the host after TB infection; therefore, the changes of weighted HRCT scores reflected the hosts' responses to the treatment on the whole.

\section{Critical indices associated with differential lung lesions between men and women with TB}

To attempt to find the factors associated with more severe lung lesions which were influenced by sex, we firstly compared a total of 48 of the physiological, inflammatory, immunological (from $\mathrm{CBC}$ ) and coagulation-associated clinical indices between the two groups, taking the $\mathrm{CBC}$ data from healthy men and women as baselines.

In healthy controls, men and women were mostly different in RBC-associated indices, with different ranges of normal values in $\mathrm{RBC}$ counts and $\mathrm{HCT}$, and
HGB levels; similarly, the values of these indices from men and women with TB were significantly different (Additional file 2: Figure S1).

There were significant differences between men and women in the counts of lymphocytes, monocytes and white blood cells in the healthy group. The differences of indices between men and women with $\mathrm{TB}$ were shown in Fig. 1 and Additional file 3: Figure S2.

Multivariate binary logistic regression (forward stepwise) was carried out to find out which differences in indices between men and women with TB were associated with severity of lung lesions. Although the indices which were selected in the models were different in separate male or female groups, and in combination, we found that similar sets of coagulation and platelet indices were involved in association with severity of male (MPV, AT3, FIB with sputum bacterial grade, PCT with cavity) and female (PT with cavity) patients with $\mathrm{TB}$, and in combination of the groups (APTT with sputum bacterial grade, PCT with cavity) (Tables 4 and 5).

In univariate regression analysis, higher grades of sputum bacterial counts were associated with increased incidence of cavities. As in a previous report [17], male 
Table $2 \mathrm{CT}$ findings of study patients

\begin{tabular}{|c|c|c|c|}
\hline \multirow[t]{2}{*}{$C T$ findings } & \multicolumn{2}{|c|}{$\begin{array}{l}\text { Prevalence of CT finding, } \\
\text { no. (\%) }\end{array}$} & \multirow[t]{2}{*}{$\begin{array}{l}P \\
\text { value }^{b}\end{array}$} \\
\hline & $\begin{array}{l}\text { Female } \\
(n=94)\end{array}$ & $\begin{array}{l}\text { Male } \\
(n=95)\end{array}$ & \\
\hline Centrilobular nodules & $93(98.94)$ & $94(98.95)$ & 1.000 \\
\hline Micronodules & $91(96.81)$ & $93(97.89)$ & 0.682 \\
\hline Bronched nodule & $88(93.62)$ & $89(93.68)$ & 0.985 \\
\hline Miliary nodule & $1(1.06)$ & $0(0.00)$ & 0.497 \\
\hline Tree in bud & $86(91.49)$ & $84(88.42)$ & 0.483 \\
\hline Cavity, number (\%) & $35(37.2)$ & $67(70.5)$ & $<0.001^{\mathrm{a}}$ \\
\hline Thin-walled cavity & $1(1.10)$ & $1(1.10)$ & - \\
\hline Thick-walled cavity & $32(34.00)$ & $58(61.10)$ & - \\
\hline Both thin- and thick-walled cavity & $2(2.10)$ & $8(8.40)$ & - \\
\hline Aspergillosis & $0(0.00)$ & $3(3.16)$ & 0.246 \\
\hline Bronchial wall thickening & $33(35.11)$ & $43(45.26)$ & 0.154 \\
\hline Bronchiectasis & $27(28.72)$ & $38(40.00)$ & 0.103 \\
\hline Bronchial impaction & $45(47.87)$ & $49(51.58)$ & 0.610 \\
\hline Emphysema (lobular) & $1(1.06)$ & $9(9.47)$ & 0.018 \\
\hline Cicatricial emphysema & $1(1.06)$ & $15(15.79)$ & 0.001 \\
\hline Bullae & $0(0.00)$ & $5(5.26)$ & 0.059 \\
\hline Ground glass opacity & $40(42.55)$ & $29(30.53)$ & 0.086 \\
\hline Consolidation & $59(62.77)$ & $60(63.16)$ & 0.956 \\
\hline Atelectasis & $6(6.40)$ & $1(1.10)$ & 0.065 \\
\hline Calcification & $33(35.10)$ & $36(7.90)$ & 0.691 \\
\hline Mediastinal lymphadenopathy & $13(13.80)$ & $20(21.10)$ & 0.191 \\
\hline Hilar lymphadenopathy & $9(9.60)$ & $18(18.90)$ & 0.066 \\
\hline Bands (parenchymal) & $20(21.30)$ & $37(38.90)$ & 0.008 \\
\hline Bronchiovascular distortion & $7(7.40)$ & $20(21.10)$ & 0.008 \\
\hline Pleural thickening & $60(63.80)$ & $74(77.90)$ & 0.033 \\
\hline Pleural effusion & $3(3.20)$ & $4(4.20)$ & 1.000 \\
\hline
\end{tabular}

sex is a predisposing factor for higher grades of sputum bacterial counts and presence of cavities, independent of age and BMI (Fig. 2 and Additional file 1: Table S3-S6). Besides, lower lymphocyte counts were associated with increased sputum bacterial counts and presence of cavities, independent of sex, age and BMI.

Other indices associated with both sputum bacterial counts and cavities were monocyte to lymphocyte ratio, IgA and TRF levels, and platelet- and coagulation-associated indices: MPV, PT, INR and FIB. These associations were influenced by adjustment with sex or age and BMI, separately or in combination. The association between platelet and coagulation indices with either cavity (MPV, FIB and INR) or cavity-associated sputum bacterial grade
Table 3 HRCT scores in men and women with TB at differential time point of anti-TB treatment

\begin{tabular}{llll}
\hline & & Female $^{\dagger}(n=15)$ & Male $^{\dagger}(n=7)$ \\
\hline HRCT scores & T0 & $16.8(11.23,22.37)$ & $25.86(15.19,36.53)$ \\
& T1 & $13.53(8.45,18.62)$ & $23.86(14.8,32.91)$ \\
& T3 & $10.00(4.89,15.11)$ & $18.71(10.24,27.19)$ \\
& $P$ value & $<0.001$ & 0.001 \\
Weighted HRCT & T0 & $38.13(23.51,52.75)$ & $48.71(23.48,73.95)$ \\
scores & T1 & $32.20(17.48,46.92)$ & $52.43(30.16,74.69)$ \\
& T3 & $23.33(9.54,37.13)$ & $41.57(17.28,65.86)$ \\
& $P$ value $^{\mathrm{a}}$ & 0.003 & 0.295 \\
& $P$ value $^{\mathrm{b}}$ & 0.158 & \\
\hline
\end{tabular}

HRCT high-resolution computed tomography, TO time of registration in SPH and before anti-TB treatment, $T 1$ about 1 month after T0, T3 about 3 months after T0

${ }^{\dagger}$ Data are displayed as means and lower and upper bounds of $95 \%$ confidence interval

${ }^{a}$ ANOVA tests with single-factor repeated measures compare the difference of scores at T0, T1 and T3 in female or male TB patients

${ }^{\mathrm{b}} \mathrm{ANOVA}$ tests with multiple-factor repeated measures compare the changes of scores at T0, $\mathrm{T} 1$ and $\mathrm{T} 3$ between female and male TB patients

Italicized numbers indicate a $P$ value of $<0.05$

(PT) was mostly influenced by sex adjustment (Fig. 2 and Additional file 1: Table S3-S6).

\section{Discussion}

Based on comparison of the severity of lung lesions between TB with diabetes and TB without diabetes, our previous study demonstrated that men with TB are associated with larger areas of necrosis in granuloma, and haemostasis indices signified exacerbated lung lesions, even at the early stage of TB [17]. To exclude the potential influence of the indices with men's tendency, e.g. smoking or other confounding factors in the previous research, this study was carried out with well-matched non-smoking men and women with primary TB without comorbidities. Our study provides clinical evidence that men are associated with more cavities and higher sputum bacterial counts than women at the time of first TB registration [18].

Previous reports indicated that severe TB correlates with neutrophil abundance and lymphocyte deficiency $[19,20]$. In our study, lower counts of peripheral lymphocytes were associated with both higher sputum bacterial loads and cavity in primary TB. Peripheral neutrophils, which were associated with sputum bacteria counts in the study involving more severe TB cases (e.g. TB with diabetes comorbidity) [17], were not associated with either cavity or sputum bacterial counts in this study.

As we have simplified our study by excluding patients with confounding factors, like smoking [21], that may influence the pathogenesis of $\mathrm{TB}$, the significant difference 


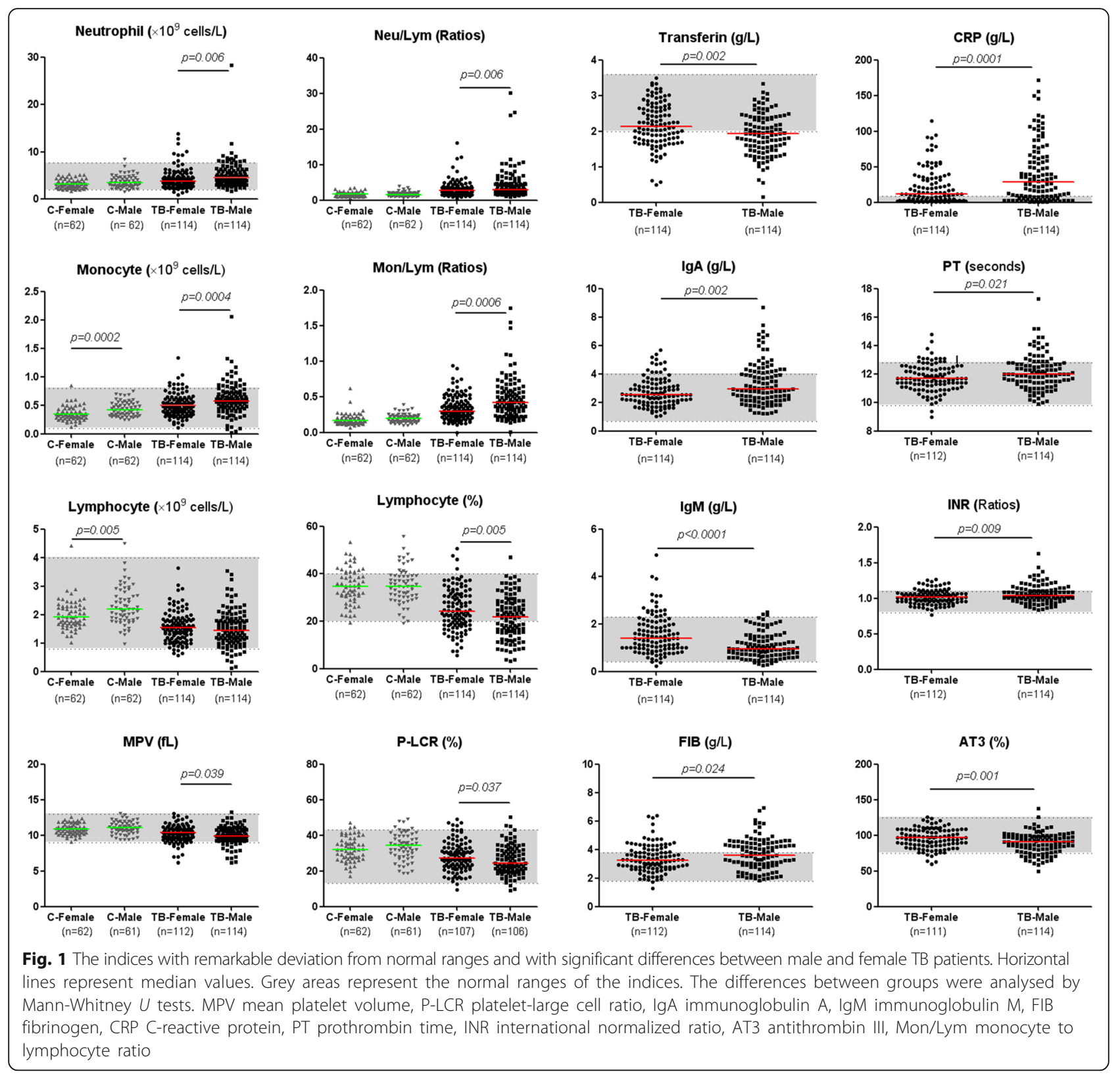

between men and women in the severity of TB-induced lung lesions may reflect the overall influences of differential sexual hormones, sex-related genetic backgrounds, genetic regulation and metabolism on the basic immune response and susceptibility to infection of $M t b$.

Women have a better ability to clear pathogens [22], and sex steroid immunomodulation has been linked to higher infection rates in men. Androgens influence gut microbiota [23], which may provoke host susceptibility to TB [24]. Estrogen promotes and testosterone downregulates T-helper (Th)1 cells, macrophage activation and antibody-dominated responses $[8,25]$, which are considered to play important roles in tuberculosis immune responses [26].
However, in the T-SPOT.TB and serum anti-TB IgG tests, similar ratios of cases with positive response were found in male and female patients. These results indicated that there were no significant differences in the frequency of TB antigen (ESAT-6, CFP-10)-specific memory $\mathrm{T}$ cells and $\mathrm{TB}$ antigen (lipoarabinomannan and 38KD antigen)-specific antibody responses between the groups; the latter two might not be the main contributors for male bias in TB pathogenesis either.

Although we did not have all information about the baseline differences in all the indices between men and women, regression analysis may give indications for the associated factors with extensive lung injury in man with $\mathrm{TB}$ on the whole, which include the associated factors 
Table 4 Summary of the variables associated with sputum bacterial counts in the models derived from multivariate regression analysis with 48 indices and cases with only men $(n=114)$ or women $(n=114)$ or in combination $(n=228)$

\begin{tabular}{|c|c|c|c|c|c|c|c|c|c|c|c|c|}
\hline \multirow{3}{*}{$\begin{array}{l}\text { Variables in } \\
\text { the equation }\end{array}$} & \multirow{2}{*}{\multicolumn{3}{|c|}{ Sig. }} & \multirow{2}{*}{\multicolumn{3}{|c|}{ OR }} & \multicolumn{6}{|c|}{$95 \% \mathrm{Cl}$ for OR } \\
\hline & & & & & & & \multicolumn{3}{|l|}{ Lower } & \multicolumn{3}{|l|}{ Upper } \\
\hline & Combined & Male & Female & Combined & Male & Female & Combined & Male & $\overline{\text { Female }}$ & Combined & Male & Female \\
\hline$\overline{\text { Sex }(\text { male })^{a}}$ & 0.006 & / & / & 3.717 & I & / & 1.445 & / & / & 9.524 & / & / \\
\hline BMI & / & 0.012 & / & / & 2.168 & / & / & 1.186 & / & / & 3.965 & / \\
\hline GLU (mmol/L) & 0.052 & / & / & 2.447 & / & / & 0.992 & / & I & 6.031 & / & / \\
\hline HistoryofTB & 0.042 & 0.086 & / & 0.000 & 0.000 & / & 0.000 & 0.000 & / & 0.000 & 0.000 & / \\
\hline HistoryofTB(1) & 0.186 & 0.997 & / & 0.308 & 0.986 & / & 0.054 & 0.001 & / & 1.767 & 1653.893 & / \\
\hline HistoryofTB(2) & 0.622 & 0.518 & / & 1.498 & 12.574 & / & 0.301 & 0.006 & / & 7.454 & $26,894.141$ & / \\
\hline HistoryofTB(3) & 0.142 & 0.912 & / & 0.191 & 0.645 & / & 0.021 & 0.000 & / & 1.739 & 1524.939 & / \\
\hline HistoryofTB(4) & 0.401 & 0.139 & / & 0.345 & 0.001 & / & 0.029 & 0.000 & / & 4.140 & 8.486 & / \\
\hline $\operatorname{Lym}\left(\times 10^{9} / \mathrm{L}\right)$ & 0.000 & 0.003 & / & 0.083 & 0.002 & / & 0.024 & 0.000 & / & 0.290 & 0.120 & / \\
\hline $\mathrm{MCHC}(\mathrm{g} / \mathrm{L})^{c}$ & 0.019 & / & / & 1.062 & / & / & 1.010 & / & / & 1.117 & / & / \\
\hline $\operatorname{ESR}(\mathrm{mm} / \mathrm{h})^{c}$ & 0.000 & / & 0.023 & 1.036 & / & 1.023 & 1.017 & / & 1.003 & 1.055 & I & 1.043 \\
\hline APTT (s) & 0.009 & / & / & 1.141 & / & / & 1.033 & / & / & 1.260 & / & / \\
\hline AT3 (\%) & / & 0.020 & / & I & 0.915 & / & / & 0.849 & / & / & 0.986 & / \\
\hline $\mathrm{FIB}(\mathrm{g} / \mathrm{L})$ & / & 0.002 & / & / & 15.968 & / & / & 2.879 & / & / & 88.572 & / \\
\hline MPV (fL) & / & 0.014 & / & / & 0.181 & / & / & 0.046 & / & / & 0.709 & / \\
\hline $\operatorname{TBAb}(+)^{\mathrm{b}}$ & / & 0.006 & / & / & 0.013 & / & / & 0.001 & / & / & 0.283 & / \\
\hline
\end{tabular}

$B M I$ body mass index, GLU fasting glucose, Lym counts of lymphocytes, MCHC mean corpuscular hemoglobin concentration, ESR erythrocyte sedimentation rate, APTT activated partial thromboplastin time, AT3 antithrombin III, FIB fibrinogen, MPV mean platelet volume, TBAb anti-TB antibody response

ORs are calculated from binary logistic analysis (forward stepwise, conditional); italicized numbers indicate a $P$ value of $<0.05$

${ }^{\mathrm{a}}$ Female as reference

${ }^{\mathrm{b}}$ Cases with negative response as reference

'Indices with $P<0.05$, but the value of OR ratio $(95 \% \mathrm{Cl})$ includes/ is close to 1.000

Table 5 Summary of the variables associated with cavity in the models of multivariate regression analysis with 48 indices and cases with only men $(n=114)$ or women $(n=114)$ or in combination $(n=228)$

\begin{tabular}{|c|c|c|c|c|c|c|c|c|c|c|c|c|}
\hline \multirow{3}{*}{$\begin{array}{l}\text { Variables in the } \\
\text { equation }\end{array}$} & \multirow{2}{*}{\multicolumn{3}{|c|}{ Sig. }} & \multirow{2}{*}{\multicolumn{3}{|c|}{ OR }} & \multicolumn{6}{|c|}{$\underline{95 \% \mathrm{Cl} \text { for } \mathrm{OR}}$} \\
\hline & & & & & & & \multicolumn{3}{|l|}{ Lower } & \multicolumn{3}{|l|}{ Upper } \\
\hline & Combined & Male & Female & Combined & Male & Female & Combined & Male & Female & Combined & Male & Female \\
\hline $\mathrm{BMI}$ & l & 0.004 & / & / & 0.516 & / & l & 0.330 & / & / & 0.807 & l \\
\hline SputumSmear (1+) & 0.012 & / & / & & / & / & & / & / & & / & / \\
\hline SputumSmear(2+) & 0.637 & / & / & 1.308 & / & / & 0.430 & / & / & 3.981 & / & / \\
\hline SputumSmear(3+) & 0.012 & / & / & 5.913 & / & / & 1.480 & / & / & 23.621 & / & / \\
\hline SputumSmear(4+) & 0.007 & / & / & 26.806 & / & / & 2.468 & / & / & 291.155 & / & / \\
\hline RDWCV & 0.001 & / & / & 2.509 & / & / & 1.464 & / & / & 4.302 & / & / \\
\hline HGB (g/L) & / & / & 0.009 & / & / & 0.928 & / & / & 0.877 & / & / & 0.981 \\
\hline $\mathrm{CRP}(\mathrm{mg} / \mathrm{L})^{\mathrm{a}}$ & 0.000 & / & / & 1.043 & / & / & 1.020 & / & / & 1.068 & / & / \\
\hline $\operatorname{ESR}(\mathrm{mm} / \mathrm{h})^{\mathrm{a}}$ & / & 0.010 & / & / & 1.076 & / & / & 1.018 & / & / & 1.138 & / \\
\hline PCT (\%) & 0.000 & 0.004 & / & 0.000 & 0.000 & / & 0.000 & 0.000 & / & 0.000 & 0.000 & / \\
\hline $\mathrm{PT}(\mathrm{s})$ & / & / & 0.046 & / & / & 2.242 & / & / & 1.016 & / & / & 4.951 \\
\hline C4 (g/L) & / & 0.016 & / & / & $1.34 \mathrm{E}+10$ & / & / & 75.786 & / & / & $2.38 \mathrm{E}+18$ & / \\
\hline $\operatorname{TBAb}(+)^{\mathrm{b}}$ & 0.008 & / & / & 4.372 & / & / & 1.469 & / & / & 13.010 & / & / \\
\hline
\end{tabular}

$B M I$ body mass index, RDW-CV red blood cell distribution width-coefficient of variation, $H G B$ haemoglobin, CRP C-reactive protein, ESR erythrocyte sedimentation rate, $P C T$ plateletcrit, $P T$ prothrombin time, $C 4$ complement component 4, TBAb anti-TB antibody response

ORs are calculated from binary logistic analysis (forward stepwise, conditional); italicized numbers indicated a $P$ value of $<0.05$

andices with $P$ value $<0.05$ but OR ratio close to 1

b Indices with $P<0.05$, but the value of OR ratio $(95 \% \mathrm{Cl})$ includes/is close to 1.000 


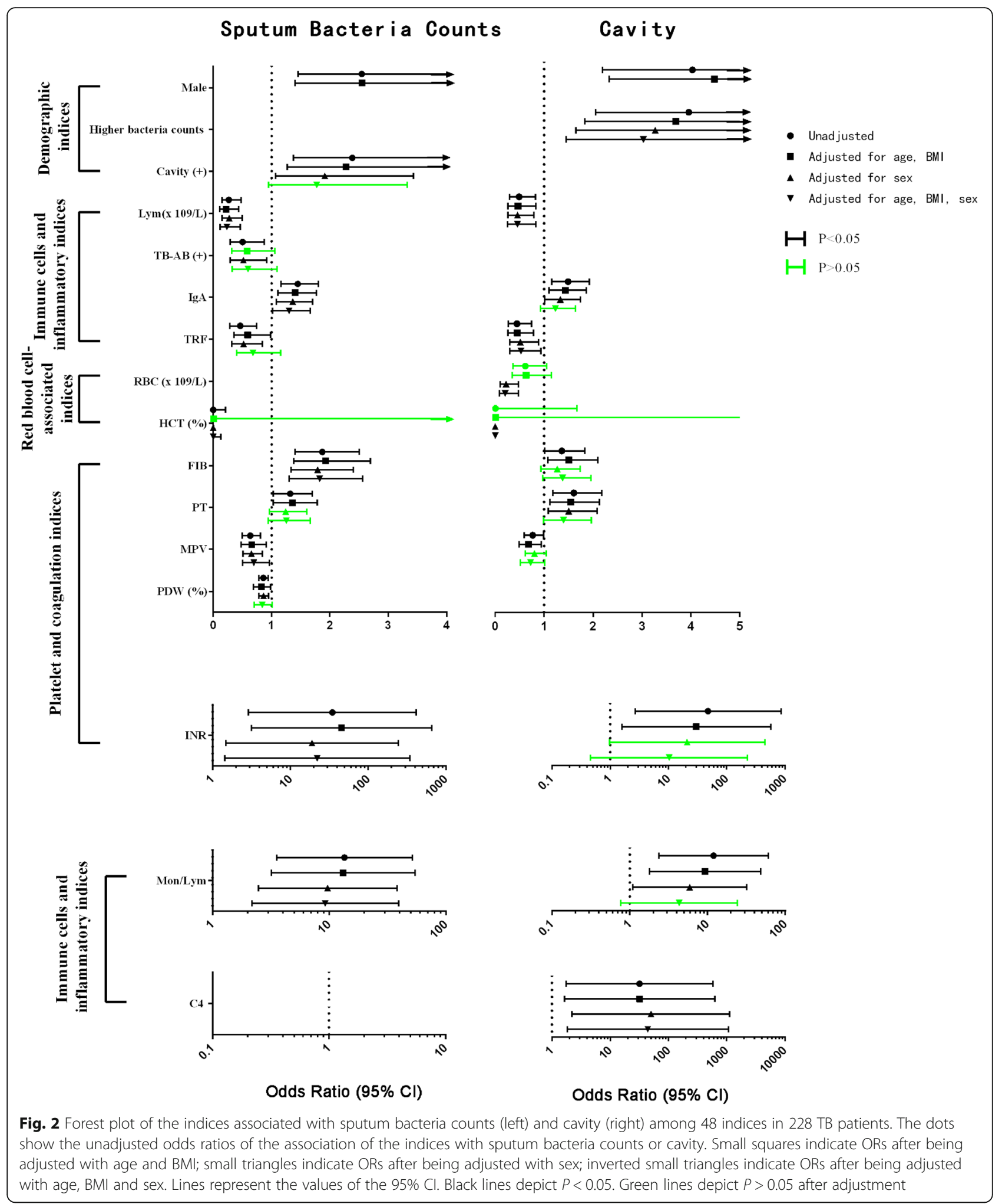

with sex bias derived from either differential physical background or differential response to TB infection.

Among all the 48 physiological, immunological and inflammatory indices, platelet and coagulation indices are identified as the associated cluster of indices with the lung lesions from both the univariate and multivariate logistic regressions, with either separate or combination cases of men and women. Consistently, the clinical manifestations 
of TB included systemic hypercoagulable states [27], and severe pulmonary TB was complicated by deep vein thrombosis in some cases [28]. Our previous study revealed that activation of coagulation and platelets (shortened PT and increased PDW) is associated with aggravated caseous necrosis and concomitant severe fibroplasia in granulomas, even at the early stage of TB pathogenesis (TB patients with negative sputum smear results and restricted lung lesion area) [17]. In this study, however, in TB cases with positive sputum smear results, increased PT and PT-associated INR are associated with more severe lung lesions (higher grades of sputum bacterial counts and presence of cavities). Similar reversion of the association in patients at different stages of TB disease also occurs between platelet indices (MPV and PDW) and the lung lesions [17]. Dynamic activation of the platelets and coagulation pathway occurs with TB infection. Increased INR and PT and reduced MPV and PDW values may reflect the exhaustion of the peripheral coagulation and platelets in patients with open lesions during the host combating with $M t b$. infection. Consistently, recent research indicated that platelets drive a proinflammatory, tissue-degrading phenotype in TB [29]. The results not only substantiate the hypothesis that platelets and coagulation pathway play important roles in TB infection, but also indicate that the association between these haemostasis indices and lung lesions is influenced mostly by sex.

Although there is a dearth of information regarding the mechanism of the sexual dimorphism of the incidence and outcome of the coagulation-associated disease (e.g. stroke) [30], it is known that genes coding for coagulation factors VIII and IX are located on chromosome $\mathrm{X}$; moreover, gonadal hormone exposure can impact coagulation and fibrinolysis [30]. Reactivity of human platelets may be influenced by sex and sex hormones, as both men and women express estrogen receptors on their platelets [31]. Some but not all reports [32] show that testosterone may activate [33] while progesterone and 17-beta estradiol may inhibit [34] aggregation of human platelets. However, differential basal expression of these coagulation factors between men and women and differential active status of the coagulation regulated by sex hormones during infection may result in different immune response and susceptibility and therefore sex bias in TB pathogenesis.

The lesser severity of TB lesions in women than in men may not only explain the male bias in the incidence of TB, but also correlate with a longer delay in seeking hospital treatment in female TB patients as shown in our investigation and in a previous report [35]. The sex bias in TB pathogenesis may also correlate with delayed recovery responses to anti-TB treatment in male patients. Therefore, studies on sex-associated disparities, especially on infection-correlated activation of coagulation and other critical modulating mechanisms involved in TB pathogenesis, may help to better understand the heterogeneity that is intrinsic to TB at the population level [36]; these studies will be crucial for better adaptation of future intervention strategies at the community level [18] and to inform the development of intervention strategies for severe cases of TB in male patients.

We should point out some of the shortcomings in this study. We have no precise information of the socioeconomic background and therefore nutritional status of patients before their registration; the latter is a key factor which influences the infected person progressing from latent TB to active TB [37]. However, as there were no statistical differences between men and women both in their BMI indices and in their ratio of cases with malnutrition (cases with BMI < 18.5), we therefore regard that the nutritional status between men and women with $\mathrm{TB}$ are similar and therefore have no critical impact on the sex bias in the pathogenesis of TB. Another shortcoming is that, although we have found that the association of the haemostasis with the lung lesions in TB is mostly influenced by sex, we still do not know the precise underlying molecular mechanism. Moreover, although there is no association of counts of peripheral neutrophils with lung lesions, difference in counts of neutrophil was found between male and female TB patients. Therefore, we cannot exclude the possibility of differential levels of neutrophil-derived soluble factors, such as matrix metalloproteinases [38, 39], driving differential cavity formation in male and female TB patients. Works based on mouse models may help to clarify the critical factor(s) involved in the sex bias in TB pathogenesis.

Furthermore, in multivariate analysis of independent associated indices for lung lesions, higher sputum bacterial counts are associated with cavitation, but not vice versa. Our result is consistent with a recent report that in those cases without cavitation, the radiological severity of disease on chest X-ray prior to treatment in smear-positive pulmonary TB patients has no association with the bacterial burden [40]. It may therefore explain the seemingly paradox association of BMI [41-43] and anti-TB antibody $[26,44,45]$ response with the bacterial counts and cavity in TB patients, in our data and previous reports.

\section{Conclusions}

Our study provides strong evidence that men with primary $\mathrm{TB}$ have significantly more severe lung lesions than women and that differential basal or infection-induced coagulation dysfunction may be involved in TB pathogenesis.

\section{Perspectives and significance}

Our findings that the association between coagulation and lung injury is influenced by gender not only suggest new mechanisms for the pathogenesis of tuberculosis, 
but also provide new insights into the biological basis of male-biased tuberculosis and other infectious diseases. An in-depth understanding of the role of the coagulation pathway in the development of these gender-biased diseases will facilitate our precise treatment and preventive measures for these diseases.

\section{Additional files}

Additional file 1: Table S1. Time to negative conversion of sputum bacteria in female and male patients with TB. Table S2. Changes of CT findings in men and women with TB after differential time of anti-TB treatment. Table S3. Association of immune, biochemical indices and CT findings with sputum bacteria counts in 228 TB patients. Table S4. Association of immune, biochemical indices with cavity in 228 TB patients. Table S5. Association of immune, biochemical indices and CT findings with high sputum smear (3+ and 4+) in 228 TB patients. Table S6. Association of immune, biochemical indices with cavity in 228 TB patients. (DOCX $70 \mathrm{~kb}$ )

Additional file 2: Figure S1. Levels of red blood cell-associated indices in male and female healthy control and TB patients. Horizontal lines represent median values. Grey areas represent the normal ranges of the index in women; the area between dashed lines represent the normal ranges of the index in men. The differences between groups were analysed by Mann-Whitney $U$ tests. RBC, red blood cell; HGB, haemoglobin; $H C T$,

haematocrit; MCHC, mean corpuscular hemoglobin concentration. (TIF $2118 \mathrm{~kb}$ )

Additional file 3: Figure S2. The indices with no or negligible differences between male and female TB patients. Horizontal lines represent median values. Grey areas represent the normal ranges of the indices. The differences between groups were analysed by Mann-Whitney $U$ tests. WBC, white blood cell; PCT, plateletcrit; APTT, activated partial thromboplastin time; $\mathrm{C}$, complement 3 ; $\mathrm{C} 4$, complement 4; FDP, fibrinogen degradation product; lgG, immunoglobulin G. (TIF $7818 \mathrm{~kb}$ )

\section{Abbreviations}

AFB: Acid-fast bacilli; BMI: Body mass index; CBC: Complete blood count; FIB: Fibrinogen; HCT: Haematocrit; HRCT: High-resolution computed tomography; INR: International normalized ratios; MPV: Mean platelet volume; PCT: Plateletcrit; PDW: Platelet distribution width; PT: Prothrombin time; TB: Tuberculosis; TRF: Transferrin

\section{Funding}

This work was supported by grants from the National Natural Science Foundation of China (grant nos. 81172806, 81471563, 81570007, 81771692, 81760578) and Natural Science Foundation of Guizhou Province (no. [2010] 3154).

\section{Availability of data and materials}

All data are presented in the paper and additional files.

\section{Authors' contributions}

YF and JS designed the research and wrote the article. JS and ASL analysed and interpreted the radiological data. WT, YC, WX, FC, WS, JH, GY, LQ, JW and $\mathrm{XH}$ collected the clinical data. YF interpreted the clinical data. JZ, WT and $Y C$ analysed the data. All authors read and approved the final manuscript.

\section{Ethics approval and consent to participate}

This study was conducted in accordance with the amended Declaration of Helsinki and the ethical guidelines of the Institutional Review Board of Tongji University (project approval number: 2014fk10). All participants gave written consent for the use of their clinical information for research purposes. Clinical data were anonymized.

\section{Consent for publication}

Not applicable

\section{Competing interests}

The authors declare that they have no competing interests.

\section{Publisher's Note}

Springer Nature remains neutral with regard to jurisdictional claims in published maps and institutional affiliations.

\section{Author details}

'Shanghai Key Laboratory of Tuberculosis, Shanghai Pulmonary Hospital, Tongji University School of Medicine, 507 Zhengmin Road, Shanghai 200433, China. ${ }^{2}$ Department of Radiology, Shanghai Pulmonary Hospital, Tongji University School of Medicine, Shanghai 200433, China. ${ }^{3}$ Department of Clinical Laboratory Medicine, Shanghai Pulmonary Hospital, Tongji University School of Medicine, Shanghai 200433, China. ${ }^{4}$ Key Laboratory of Environment Pollution Monitoring and Disease Control, Ministry of Education, School of Public Health, Guizhou Medical University, Guiyang 550025, Guizhou, China. ${ }^{5}$ Department of Epidemiology and Biostatistics, Tongji University School of Medicine, Shanghai 200433, China. ${ }^{6} \mathrm{Clinic}$ and Research Centre of Tuberculosis, Shanghai Pulmonary Hospital, Tongji University School of Medicine, Shanghai 200433, China.

Received: 31 July 2018 Accepted: 24 September 2018

Published online: 10 October 2018

\section{References}

1. WHO. Global tuberculosis report 2017. Geneva: World Health Organization; 2017.

2. WHO. Global tuberculosis report 2018. Annex 2: Country profiles for 30 high TB burden countries. Geneva: World Health Organization; 2018.

3. Weiss MG, Sommerfeld J, Uplekar MW. Social and cultural dimensions of gender and tuberculosis. Int J Tuberc Lung Dis. 2008;12:829-30.

4. Mason PH, Degeling C, Denholm J. Sociocultural dimensions of tuberculosis: an overview of key concepts. Int J Tuberc Lung Dis. 2015;19:1135-43.

5. Borgdorff MW, Nagelkerke NJ, Dye C, Nunn P. Gender and tuberculosis: a comparison of prevalence surveys with notification data to explore sex differences in case detection. Int J Tuberc Lung Dis. 2000;4:123-32.

6. Reardon S. Infections reveal inequality between the sexes. Nature. 2016;534:447.

7. Guerra-Silveira F, Abad-Franch F. Sex bias in infectious disease epidemiology: patterns and processes. PLoS One. 2013;8:e62390.

8. Neyrolles O, Quintana-Murci L. Sexual inequality in tuberculosis. PLoS Med. 2009;6:e1000199.

9. Dibbern J, Eggers L, Schneider BE. Sex differences in the C57BL/6 model of Mycobacterium tuberculosis infection. Sci Rep. 2017;7:10957.

10. Bini El, Mata Espinosa D, Marquina Castillo B, Barrios Payán J, Colucci D, Cruz $A F$, et al. The influence of sex steroid hormones in the immunopathology of experimental pulmonary tuberculosis. PLoS One. 2014;9:e93831.

11. Salie M, Daya M, Lucas LA, Warren RM, van der Spuy GD, van Helden PD, et al. Association of toll-like receptors with susceptibility to tuberculosis suggests sex-specific effects of TLR8 polymorphisms. Infect Genet Evol. 2015;34:221-9.

12. Cheesbrough $M$. ZN staining technique and Auramine $\mathrm{O}$ staining technique for the detection of Mycobacterium tuberculosis. In: District laboratory practice in developing countries. Part 2. (2nd ed.). Cambridge: Cambridge University Press; 2006.

13. In Chinese Anti-tuberculosis Association, ed. Standard procedures for laboratory diagnosis of tuberculosis. Beijing: Chinese Education and Culture Press; 2006. p. 16.

14. Ors F, Deniz O, Bozlar U, Gumus S, Tasar M, Tozkoparan E, et al. Highresolution $C T$ findings in patients with pulmonary tuberculosis: correlation with the degree of smear positivity. J Thorac Imaging. 2007;22:154-9.

15. Ralph AP, Ardian M, Wiguna A, Maguire GP, Becker NG, Drogumuller G, et al. A simple, valid, numerical score for grading chest $x$-ray severity in adult smear-positive pulmonary tuberculosis. Thorax. 2010;65:863-9.

16. Im JG, Itoh H, Shim YS, Lee JH, Ahn J, Han MC, et al. Pulmonary tuberculosis: $C T$ findings--early active disease and sequential change with antituberculous therapy. Radiology. 1993;186:653-60.

17. Dong Z, Shi J, Dorhoi A, Zhang J, Soodeen-Lalloo AK, Tan W, et al. Hemostasis and lipoprotein indices signify exacerbated lung injury in tuberculosis with diabetes comorbidity. Chest. 2018;153:1187-200.

18. Thorson A, Long NH, Larsson LO. Chest X-ray findings in relation to gender and symptoms: a study of patients with smear positive tuberculosis in Vietnam. Scand J Infect Dis. 2007;39:33-7.

19. Panteleev AV, Nikitina IY, Burmistrova IA, Kosmiadi GA, Radaeva TV, Amansahedov RB, et al. Severe tuberculosis in humans correlates best with 
neutrophil abundance and lymphocyte deficiency and does not correlate with antigen-specific CD4 T-cell response. Front Immunol. 2017;8:963.

20. Philips JA. Neutrophils: double agents for TB. Sci Transl Med. 2017;9(394). https://doi.org/10.1126/scitranslmed.aan6195

21. Berg RD, Levitte S, O'Sullivan MP, O'Leary SM, Cambier CJ, Cameron J, et al. Lysosomal disorders drive susceptibility to tuberculosis by compromising macrophage migration. Cell. 2016;165:139-52.

22. Klein SL, Roberts CW. Sex hormones and immunity to infection. Berlin: Springer Verlag; 2010.

23. Yurkovetskiy L, Burrows $M$, Khan AA, Graham L, Volchkov P, Becker L, et al. Gender bias in autoimmunity is influenced by microbiota. Immunity. 2013, 39:400-12.

24. Khan N, Vidyarthi A, Nadeem S, Negi S, Nair G, Agrewala JN. Alteration in the gut microbiota provokes susceptibility to tuberculosis. Front Immunol. 2016;7:529.

25. Grossman CJ. Interactions between the gonadal steroids and the immune system. Science. 1985;227:257-61.

26. Li H, Javid B. Antibodies and tuberculosis: finally coming of age? Nat Rev Immunol. 2018. https://doi.org/10.1038/s41577-018-0028-0. [Epub ahead of print]

27. Kager LM, Blok DC, Lede IO, Rahman W, Afroz R, Bresser P, et al. Pulmonary tuberculosis induces a systemic hypercoagulable state. J Inf Secur. 2015;70:324-34.

28. Turken O, Kunter E, Sezer M, Solmazgul E, Cerrahoglu K, Bozkanat E, et al. Hemostatic changes in active pulmonary tuberculosis. Int I Tuberc Lung Dis. 2002;6:927-32.

29. Fox KA, Kirwan DE, Whittington AM, Krishnan N, Robertson BD, Gilman RH, et al. Platelets regulate pulmonary inflammation and tissue destruction in tuberculosis. Am J Respir Crit Care Med. 2018;198:245-55.

30. Roy-O'Reilly M, McCullough LD. Sex differences in stroke: the contribution of coagulation. Exp Neurol. 2014;259:16-27.

31. Jayachandran M, Miller VM. Human platelets contain estrogen receptor alpha, caveolin-1 and estrogen receptor associated proteins. Platelets. 2003; 14:75-81.

32. Li S, Li X, Li J, Deng X, Li Y. Inhibition of oxidative-stress-induced platelet aggregation by androgen at physiological levels via its receptor is associated with the reduction of thromboxane $\mathrm{A} 2$ release from platelets. Steroids. 2007:72:875-80.

33. Ajayi AA, Mathur R, Halushka PV. Testosterone increases human platelet thromboxane A2 receptor density and aggregation responses. Circulation. 1995;91:2742-7.

34. Selles J, Polini N, Alvarez C, Massheimer V. Progesterone and 17 betaestradiol acutely stimulate nitric oxide synthase activity in rat aorta and inhibit platelet aggregation. Life Sci. 2001;69:815-27.

35. Thorson A, Hoa NP, Long NH. Health-seeking behaviour of individuals with a cough of more than 3 weeks. Lancet. 2000;356:1823-4.

36. Cadena AM, Fortune SM, Flynn JL. Heterogeneity in tuberculosis. Nat Rev Immunol. 2017;17:691-702.

37. Chandrasekaran P, Saravanan N, Bethunaickan R, Tripathy S. Malnutrition: modulator of immune responses in tuberculosis. Front Immunol. 2017;8:1316

38. Ong CW, Elkington PT, Brilha S, Ugarte-Gil C, Tome-Esteban MT, Tezera LB, et al. Neutrophil-derived MMP-8 drives AMPK-dependent matrix destruction in human pulmonary tuberculosis. PLoS Pathog. 2015;11:e1004917.

39. Ong CW, Elkington PT, Friedland JS. Tuberculosis, pulmonary cavitation, and matrix metalloproteinases. Am J Respir Crit Care Med. 2014;190:9-18.

40. Murthy SE, Chatterjee F, Crook A, Dawson R, Mendel C, Murphy ME, et al. Pretreatment chest $x$-ray severity and its relation to bacterial burden in smear positive pulmonary tuberculosis. BMC Med. 2018;16:73.

41. Lin HH, Wu CY, Wang CH, Fu H, Lönnroth K, Chang YC, et al. Association of obesity, diabetes, and risk of tuberculosis: two population-based cohorts. Clin Infect Dis. 2018;66:699-705.

42. Oxlade O, Murray M. Tuberculosis and poverty: why are the poor at greater risk in India? PLoS One. 2012;7:e47533.

43. Zhang $H$, Li X, Xin H, Li H, Li M, Lu W, et al. Association of body mass index with the tuberculosis infection: a population-based study among 17796 adults in rural China. Sci Rep. 2017;7:41933.

44. Achkar JM, Prados-Rosales R. Updates on antibody functions in Mycobacterium tuberculosis infection and their relevance for developing a vaccine against tuberculosis. Curr Opin Immunol. 2018:53:30-7.

45. Jacobs AJ, Mongkolsapaya J, Screaton GR, McShane H, Wilkinson RJ. Antibodies and tuberculosis. Tuberculosis (Edinb). 2016:101:102-13.

\section{Ready to submit your research? Choose BMC and benefit from:}

- fast, convenient online submission

- thorough peer review by experienced researchers in your field

- rapid publication on acceptance

- support for research data, including large and complex data types

- gold Open Access which fosters wider collaboration and increased citations

- maximum visibility for your research: over $100 \mathrm{M}$ website views per year

At BMC, research is always in progress.

Learn more biomedcentral.com/submissions 